\title{
Trends and Regional Distribution Pattern of Female Lung Cancer - China, 1992-2016
}

\author{
Xue $\mathrm{Ni}^{1}$; Bei Zhangi ${ }^{1}$; Ning Xu' ${ }^{1}$; Xinying Zeng ${ }^{2}$; Qiang Wang ${ }^{1, *}$
}

\section{Summary \\ What is already known about this topic?}

The incidence of female lung cancer in China has been rising, whereas the incidence ratio among men and women is declining. The rising trend of female lung cancer is a prominent public health concern for China. What is added by this report?

The joint secular trends distribution of the incidence, mortality, and years of life lost (YLLs) due to premature death of lung cancer in Chinese women demonstrated a certain regionality, which implied the risk factors for female lung cancer in China might be different by region. Common determinant risk factors may exist in regions sharing the same joint secular trends.

What are the implications for public health practice?

The secular trends and the regional pattern of the trends of female lung cancer in China suggested that the prevention and control of female lung cancer should be implemented with targeted regional interventions.

According to the 2018 cancer estimates from the Global Cancer Observatory (GLOBOCAN) of the World Health Organization (WHO), lung cancer was a leading cause of cancer death in China (1). The incidence of female lung cancer in China was approximately 1.5 times that of the women worldwide (1). Tobacco smoke is believed to be the leading risk factor for lung cancer with about $90 \%$ of lung cancer deaths being linked to cigarette smoking, but the current rate of smoking in Chinese women has been estimated to be around $2 \%-4 \%$ in the past 20 years. Therefore, the growing trend of lung cancer in Chinese women warrants major concern and the key risk factors have yet to be elucidated. This study aimed to explore the secular trends and regional distribution of female lung cancer in China.
The results of the Global Burden of Disease 2016 (GBD 2016) (2-3) was used in this report. Joinpoint regression analysis was applied to fit the secular female lung cancer trends of age-standardized incidence rate (ASIR), age-standardized mortality rate (ASMR), and age-standardized years of life lost due to premature death rate (ASYLLR) for the mainland of China including the 31 provincial-level administrative divisions (PLADs) from 1992-2016. Joinpoint regression models was used to divide the long-term, secular trend data into a few continuous segments by turning points, which was more reasonable for describing changes in trend data.

The World Standard Population database (WHO 2000-2025) (4) was used as the reference population in the calculation of the age-standardized rates (ASRs) of 31 PLADs. The secular trends of ASRs were analyzed using a joinpoint regression model in which the logarithm of the ASRs was used as the dependent variable and the year was used as the explanatory variable. Best-fitting points (joinpoints) were chosen when the rate changed significantly (5), and the lung cancer trend was divided into several segments. A maximum of four joinpoints were set in the regression analysis. The test of significance was performed by the Monte Carlo Permutation method. The significant level was set at $p<0.05$. Joinpoint regression models were analyzed by the Joinpoint Regression Program Version 4.5.0 (Statistical Research and Applications Branch, National Cancer Institute, Bethesda, MD, USA).

In the past 25 years for China as a whole, the secular trend of ASIR rose upward and the trend of ASMR or ASYLLR moved downwards (Figure 1). The average annual percent change (AAPC) ${ }^{*}$ of the ASIR, ASMR, and ASYLLR were $0.7 \%(p<0.05),-0.3 \%(p<0.05)$, and $-0.7 \%(p<0.05)$, respectively. However, ASIR and ASMR all showed increases before 2005, and ASIR slight decreased after increase up to 2005 while the

\footnotetext{
* AAPC: a weighted average of the APCs (Annual Percent Changes: Annual percentage changes of rate in each segment trend) from the joinpoint model with the weights equal to the length of the APC interval. This means that rates change at a constant percentage every year throughout the entire period.
} 


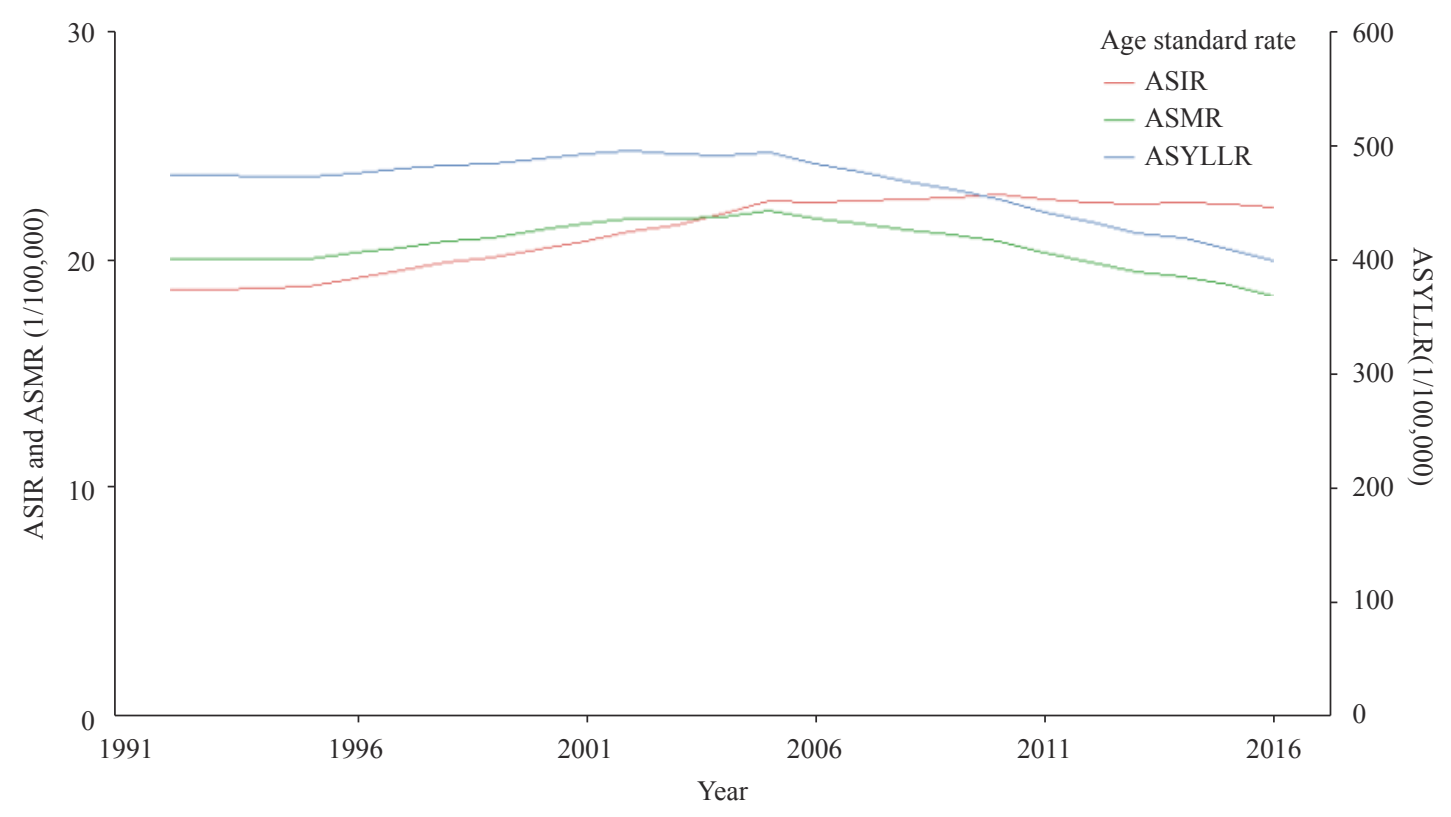

FIGURE 1. Long-term trends of the ASIR, ASMR, and ASYLLR of the female lung cancer of China. Abreviation: ASIR=agestandardized incidence rate; ASMR=age-standardized mortality rate; ASYLLR=age-standardized the years of live lost due to premature death rate.

ASMR showed a significant downward trend.

The secular trends of the ASIR, ASMR, and ASYLLR among 31 PLADs are inconsistent (Table 1) as not all ASIRs for 31 PLADs created a rising trend. The ASIR in $5(16.1 \%)$ of the PLADs remained relatively steady, while the ASIR in 9 PLADs (29.0\%) declined noticeably. The PLADs with the most rapid growing ASIR of female lung cancer were Guangxi and Sichuan with a $2 \%$ AAPC. The AAPC of ASIR among 5 PLADs including Beijing, Tianjin, Guizhou, Yunnan, and Gansu were around zero.

There were also regional differences in ASMR and ASYLLR among the 31 PLADs. The descending trends of ASMR among 23 PLADs (74.2\%) and ASYLLR among 27 PLADs $(87.1 \%)$ were consistent with trends seen nationwide. But the trends of ASMR or ASYLLR in the remaining PLADs were rising upward or stable. The greatest declines of ASMR was found in Beijing, with 2\% AAPCs of ASMR approximately. And the most rapid declines of ASYLLR were found within Beijing, Tianjin, and Inner Mongolia (2\%).

The joint secular trends of the ASIR, ASMR, and ASYLLR of female lung cancer among 31 PLADs showed 5 distinct regional patterns (Figure 2). A total of 9 PLADs in northern China had falling trends in ASIR, ASMR, and ASYLLR (Pattern A), 4 PLADs in western China had rising trends in ASIR and ASMR (Pattern B), 9 PLADs in southeastern China had trends of rising ASIR, falling ASMR, and falling
ASYLLR (Pattern C), 4 PLADs in eastern China had trends of rising ASIR, stable in ASMR, and falling ASYLLR (Pattern D), and the other 5 PLADs in southwestern China had trends of steady ASIR, falling ASMR, and falling ASYLLR (Pattern E). As shown in Pattern B, except for one province, the three other provinces had rising trends in ASYLLR. The regional linkage pattern of the joint secular trends of female lung cancer suggested that the determinant risk factors might be different by region.

\section{DISCUSSION}

We found the ASIR was rising and the ASMR or ASYLLR were falling in the past 25 years. The overall secular trends of female lung cancer in China as a whole were similar to results found by Liu et al (6). However, we know that the year of 2005 was a turning point, and the change in female lung cancer rate was the most obvious. This may be related to the enhanced management of China's Cancer Registration and Death Registration system in 2005 that intensified construction of the registration system and data quality. But it may also be related to improvements in the ecological environment and the level of medical treatment and healthcare.

Further trend analyses based on PLADs showed the joint trends among the 31 PLADs were not consistent. Although ASIR among the 17 PLADs are rising, their 
TABLE 1. National and provincial-level AAPC and female lung cancer ASRs in China, 1992-2016.

\begin{tabular}{|c|c|c|c|c|c|c|c|c|c|}
\hline \multirow{2}{*}{ Region } & \multicolumn{2}{|c|}{$\operatorname{ASIR}(1 / 100,000)$} & \multicolumn{2}{|c|}{$\operatorname{ASMR}(1 / 100,000)$} & \multicolumn{2}{|c|}{ ASYLLR $(1 / 100,000)$} & \multicolumn{3}{|c|}{ AAPC $_{\text {ASIR }}(95 \% \mathrm{Cl})$ AAPC $_{\text {ASMR }}(95 \% \mathrm{Cl})$ AAPC $_{\text {ASYLLR }}(95 \% \mathrm{Cl})$} \\
\hline & 1992 & 2016 & 1992 & 2016 & 1992 & 2016 & $1992-2016$ & $1992-2016$ & 1992-2016 \\
\hline China & 18.8 & 22.3 & 20.1 & 18.5 & 474.9 & 400.5 & $0.7(0.6,0.8)^{*}$ & $-0.3(-0.5,-0.2)^{\star}$ & $-0.7(-0.8,-0.6)^{\star}$ \\
\hline Anhui & 19.0 & 23.0 & 20.8 & 19.7 & 479.8 & 430.7 & $0.8(0.6,0.9)^{*}$ & $-0.2(-0.3,-0.1)^{\star}$ & $-0.4(-0.5,-0.4)^{*}$ \\
\hline Beijing & 26.0 & 26.4 & 25.3 & 17.2 & 571.1 & 350.0 & $0.1(-0.0,0.2)$ & $-1.6(-1.8,-1.5)^{\star}$ & $-2.0(-2.1,-1.9)^{\star}$ \\
\hline Chongqing & 23.4 & 27.7 & 24.0 & 22.5 & 579.7 & 488.1 & $0.7(0.6,0.9)^{*}$ & $-0.3(-0.5,-0.1)^{*}$ & $-0.7(-0.9,-0.6)^{*}$ \\
\hline Fujian & 14.9 & 17.0 & 16.4 & 13.7 & 386.3 & 307.5 & $0.5(0.3,0.8)^{*}$ & $-0.7(-0.9,-0.6)^{*}$ & $-0.9(-1.1,-0.8)^{*}$ \\
\hline Gansu & 9.7 & 9.6 & 10.3 & 9.6 & 240.6 & 220.0 & $-0.0(-0.2,0.1)$ & $-0.2(-0.4,-0.0)^{*}$ & $-0.3(-0.4,-0.2)^{*}$ \\
\hline Guangdong & 17.1 & 23.4 & 19.2 & 15.5 & 446.0 & 345.2 & $1.3(1.0,1.6)^{*}$ & $-0.9(-1.0,-0.7)^{\star}$ & $-1.0(-1.2,-0.9)^{*}$ \\
\hline Guangxi & 13.2 & 22.3 & 14.4 & 16.6 & 349.0 & 372.6 & $2.2(1.9,2.4)^{\star}$ & $0.6(0.5,0.8)^{*}$ & $0.3(0.1,0.5)^{*}$ \\
\hline Guizhou & 15.7 & 15.7 & 17.0 & 16.3 & 401.6 & 361.5 & $0.0(-0.2,0.2)$ & $-0.2(-0.3,-0.1)^{*}$ & $-0.4(-0.5,-0.3)^{*}$ \\
\hline Hainan & 18.1 & 16.0 & 18.8 & 15.2 & 434.1 & 335.5 & $-0.5(-0.6,-0.4)^{*}$ & $-0.9(-1.0,-0.7)^{\star}$ & $-1.1(-1.2,-0.9)^{*}$ \\
\hline Hebei & 15.9 & 21.0 & 16.8 & 16.7 & 389.0 & 365.6 & $1.2(1.1,1.2)^{\star}$ & $-0.0(-0.1,0.0)$ & $-0.3(-0.3,-0.2)^{*}$ \\
\hline Heilongjiang & 43.0 & 38.5 & 46.4 & 34.3 & 1088.7 & 727.4 & $-0.4(-0.8,-0.1)^{\star}$ & $-1.2(-1.4,-1.0)^{\star}$ & $-1.6(-1.8,-1.4)^{*}$ \\
\hline Henan & 15.1 & 17.7 & 15.8 & 17.3 & 371.7 & 366.4 & $0.7(0.5,0.8)^{\star}$ & $0.4(0.2,0.5)^{*}$ & $-0.1(-0.2,0.1)$ \\
\hline Hubei & 16.1 & 21.2 & 16.5 & 16.5 & 385.6 & 367.7 & $1.2(1.0,1.3)^{*}$ & $0.0(-0.1,0.1)$ & $-0.2(-0.3,-0.1)^{\star}$ \\
\hline Hunan & 16.1 & 17.6 & 17.3 & 15.4 & 416.2 & 345.4 & $0.4(0.3,0.5)^{*}$ & $-0.5(-0.7,-0.3)^{*}$ & $-0.8(-0.9,-0.6)^{*}$ \\
\hline Inner Mongolia & 24.1 & 21.4 & 25.7 & 17.5 & 607.7 & 377.5 & $-0.5(-0.6,-0.3)^{*}$ & $-1.6(-1.8,-1.4)^{*}$ & $-2.0(-2.1,-1.8)^{\star}$ \\
\hline Jiangsu & 17.7 & 22.3 & 19.8 & 15.9 & 449.6 & 347.5 & $1.0(0.9,1.1)^{*}$ & $-0.9(-1.0,-0.7)^{\star}$ & $-1.0(-1.2,-0.9)^{\star}$ \\
\hline Jiangxi & 16.1 & 15.4 & 17.1 & 15.4 & 412.2 & 337.0 & $-0.2(-0.4,-0.0)^{\star}$ & $-0.4(-0.6,-0.2)^{\star}$ & $-0.9(-1.0,-0.7)^{*}$ \\
\hline Jilin & 31.1 & 33.6 & 33.9 & 25.5 & 794.6 & 549.0 & $0.3(0.2,0.5)^{*}$ & $-1.1(-1.4,-0.8)^{\star}$ & $-1.5(-1.7,-1.3)^{\star}$ \\
\hline Liaoning & 30.6 & 28.0 & 33.7 & 23.9 & 787.3 & 500.2 & $-0.4(-0.5,-0.3)^{*}$ & $-1.4(-1.6,-1.3)^{\star}$ & $-1.9(-2.0,-1.8)^{*}$ \\
\hline Ningxia & 16.7 & 16.3 & 17.4 & 16.1 & 409.1 & 351.4 & $-0.1(-0.1,-0.0)^{\star}$ & $-0.3(-0.4,-0.2)^{\star}$ & $-0.6(-0.7,-0.6)^{\star}$ \\
\hline Qinghai & 13.1 & 15.4 & 13.6 & 15.6 & 323.5 & 354.4 & $0.7(0.5,0.8)^{*}$ & $0.6(0.4,0.8)^{*}$ & $0.4(0.2,0.6)^{*}$ \\
\hline Shaanxi & 14.7 & 13.9 & 16.2 & 13.3 & 381.1 & 293.9 & $-0.2(-0.4,-0.0)^{\star}$ & $-0.8(-1.0,-0.7)^{*}$ & $-1.1(-1.2,-0.9)^{*}$ \\
\hline Shandong & 21.7 & 26.9 & 23.7 & 24.2 & 553.9 & 518.0 & $0.9(0.8,1.1)^{*}$ & $0.1(-0.0,0.2)$ & $-0.3(-0.4,-0.2)^{*}$ \\
\hline Shanghai & 19.8 & 23.5 & 19.1 & 15.0 & 424.8 & 314.5 & $0.7(0.6,0.9)^{*}$ & $-1.0(-1.2,-0.8)^{\star}$ & $-1.2(-1.4,-1.1)^{\star}$ \\
\hline Shanxi & 16.0 & 18.0 & 16.5 & 17.0 & 388.2 & 359.6 & $0.5(0.4,0.7)^{*}$ & $0.1(-0.0,0.3)$ & $-0.3(-0.4,-0.2)^{*}$ \\
\hline Sichuan & 18.2 & 30.1 & 18.8 & 24.4 & 479.5 & 531.0 & $2.1(1.9,2.4)^{*}$ & $1.1(0.9,1.2)^{*}$ & $0.4(0.2,0.6)^{\star}$ \\
\hline Tianjin & 33.6 & 34.6 & 35.9 & 24.4 & 818.2 & 498.8 & $0.1(-0.2,0.4)$ & $-1.5(-1.8,-1.3)^{\star}$ & $-2.0(-2.2,-1.8)^{*}$ \\
\hline Tibet & 4.8 & 4.6 & 5.0 & 4.8 & 121.3 & 113.6 & $-0.2(-0.3,-0.0)^{\star}$ & $-0.2(-0.2,-0.1)^{\star}$ & $-0.3(-0.4,-0.2)^{\star}$ \\
\hline Xinjiang & 15.8 & 13.0 & 15.6 & 12.9 & 370.5 & 274.0 & $-0.8(-1.0,-0.6)^{\star}$ & $-0.8(-1.0,-0.6)^{\star}$ & $-1.2(-1.4,-1.1)^{*}$ \\
\hline Yunnan & 14.3 & 13.4 & 15.6 & 13.5 & 374.5 & 310.6 & $-0.3(-0.5,0.0)$ & $-0.6(-0.8,-0.4)^{\star}$ & $-0.8(-0.9,-0.6)^{*}$ \\
\hline Zhejiang & 17.2 & 21.1 & 18.5 & 16.6 & 438.4 & 358.4 & $0.9(0.7,1.0)^{*}$ & $-0.4(-0.7,-0.1)^{\star}$ & $-0.8(-1.0,-0.5)^{*}$ \\
\hline
\end{tabular}

Abreviation: AAPC=average annual percent change; ASR=age-standardized rates;ASIR=age-standardized incidence rate; ASMR=agestandardized mortality rate; ASYLLR=age-standardized years of live lost due to premature death rate.

${ }^{*}$ Represents statistical significance, $p<0.05$.

trends in ASMR and ASYLLR were different. They were in 5 patterns of joint changing trends, and the distribution of the joint trends were not chaotic or random. The PLADs sharing common patterns were found to be geographically adjacent. The linkage regional patterns suggested that the phenotypes of female lung cancer might also be different by region. There might exist different key risks of female lung cancer in different regions, such as air pollution. Chen et al. (7) also found provincial differences in the potential risks of population attributable fraction (PAF). In addition, the overall ASIR trend of female lung cancer in northeastern China was currently declining, but ASIR of these PLADs were the highest in China. Risk factors in the northeastern China need further attention. 


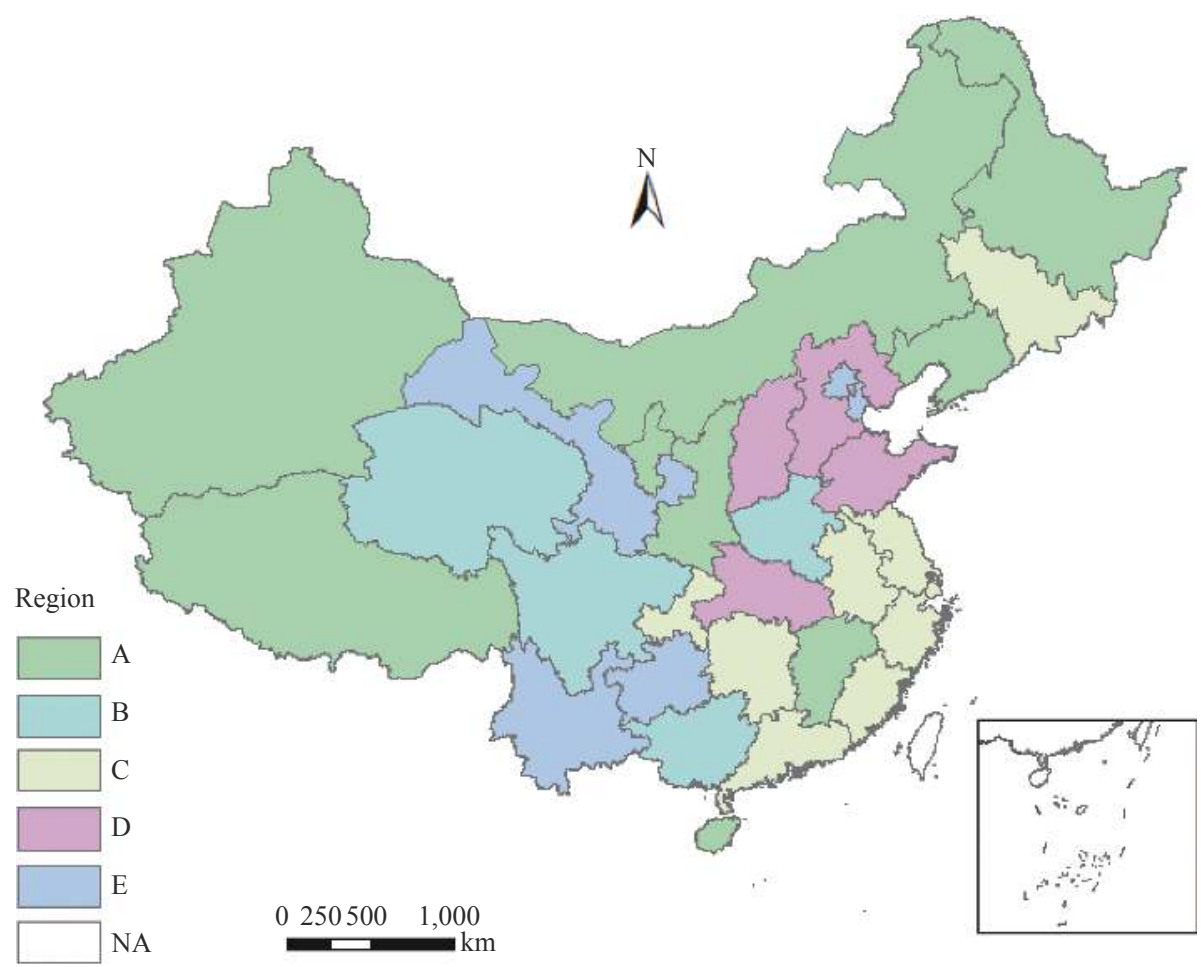

FIGURE 2. Joint changing trends of female lung cancer in China at the provincial level, 1992-2016. The pattern illustrated different average annual percent change (AAPC) trends in combination of age standardized incidence rate (ASIR) and age standardized mortality rate (ASMR), and age-standardized years of life lost due to premature death rate (ASYLLR). Pattern A, significant fall in ASIR, ASMR, and ASYLLR; Pattern B, significant rise in ASIR, ASMR, and ASYLLR; Pattern C, significant rise in ASIR, significant fall in ASMR and in ASYLLR; Pattern D, significant rise in ASIR, stable in ASMR, and significant fall in ASYLLR; Pattern E, stable in ASIR, significant fall in ASMR, and significant fall in ASYLLR; NA, no data was available.

Aging is believed to be closely correlated with lung cancer (8-9). Among the 5 joint changing patterns, the trends of ASMR rates among 4 PLADs in western China kept rising, which differed from that of the falling trends in other 27 PLADs. This suggests that aging was not the key risk for the secular rising trends of female lung cancer among 4 PLADs. The risk factors of female lung cancer in western China need to be further explored.

The incidence and mortality extracted from GBD data in this report might differ slightly from cancer registration data. However, GBD data was obtained by analyzing data from multiple sources using a uniform and comparable method, which increased its representativeness.

There were some limitations in this study. First, due to the availability and quality of cancer registration data in China, the data scale of this study was only limited to the provincial level, and the trends of prevalence of female lung cancer could not be studied at a more sophisticated scale. Second, this study inferred that the tendency of female lung cancer and its risk factors might be aggregated based on the characteristics of the trend of female lung cancer, and further studies are required.

In summary, the linkage regional pattern of joint trends in incidence, mortality, and ASYLLR suggested there might exist region-specific risks of female lung cancer. A region-specific strategy might be necessary for prevention and control of female lung cancer in China, especially for the Pattern B region where the ASIR increased rapidly, which may be caused by new risk factors. The increase in ASMR and ASYLLR suggested that early detection and early treatment may be very important.

Conflicts: No potential conflicts of interest were disclosed.

doi: $10.46234 / \mathrm{ccdcw} 2020.166$

\#Corresponding author: Qiang Wang, wangqiang@nieh.chinacdc.cn.

\footnotetext{
${ }^{1}$ National Institute of Environmental Health, Chinese Center for Disease Control and Prevention, Beijing, China; ${ }^{2}$ The National Center for Chronic and Noncommunicable Disease Control and Prevention, Chinese Center for Disease Control and Prevention, Beijing, China.
}

Submitted: March 05, 2020; Accepted: August 01, 2020 


\section{REFERENCES}

1. Bray F, Ferlay J, Soerjomataram I, Siegel RL, Torre LA, Jemal A. Global cancer statistics 2018: GLOBOCAN estimates of incidence and mortality worldwide for 36 cancers in 185 countries. CA: A Cancer J Clin 2018;68(6):394 - 424. http://dx.doi.org/10.3322/caac.21492.

2. GBD 2016 Disease and Injury Incidence and Prevalence Collaborators. Global, regional, and national incidence, prevalence, and years lived with disability for 328 diseases and injuries for 195 countries, 1990-2016: a systematic analysis for the Global Burden of Disease Study 2016. Lancet 2017;390(10100):1211 - 59. http://dx.doi.org/10.1016/S0140-6736 (17)32154-2.

3. Zhou MG, Wang HD, Zhu J, Chen WQ, Wang LH, Liu SW, et al. Cause-specific mortality for 240 causes in China during 1990-2013: a systematic subnational analysis for the Global Burden of Disease Study 2013. Lancet 2016;387(10015):251 - 72. http://dx.doi.org/10.1016/ S0140-6736(15)00551-6.

4. World Health Organization (WHO). World (WHO 2000-2025) standard. https://seer.cancer.gov/stdpopulations/world.who.html.[202008-01].
5. Kim HJ, Fay MP, Feuer EJ, Midthune DN. Permutation tests for joinpoint regression with applications to cancer rates. Stat Med 2000;19(3):335 - 51. http://dx.doi.org/10.1002/(SICI)1097-0258 (20000215) 19:3<335::AID-SIM336>3.0.CO;2-Z.

6. Liu YN, Liu JM, Yin P, Liu SW, Cai Y, You JL, et al. The disease burden of malignant tumor in China, 1990 and 2010. Chin J Prev Med 2015;49(4):309 - 14. http://dx.doi.org/10.3760/cma.j.issn.0253-9624. 2015.04.006. (In Chinese).

7. Chen WQ, Xia CF, Zheng RS, Zhou MG, Lin CQ, Zeng HM, et al. Disparities by province, age, and sex in site-specific cancer burden attributable to 23 potentially modifiable risk factors in China: a comparative risk assessment. Lancet Glob Health 2019;7(2):e257 - 69 http://dx.doi.org/10.1016/S2214-109X(18)30488-1.

8. Wingo PA, Cardinez CJ, Landis SH, Greenlee RT, Ries LAG, Anderson $\mathrm{RN}$, et al. Long-term trends in cancer mortality in the United States, 1930-1998. Cancer 2003;97(S12):3133 - 275. http://dx.doi.org/10. $1002 /$ cncr. 11380 .

9. Blanchard EM, Arnaoutakis K, Hesketh PJ. Lung cancer in octogenarians. J Thorac Oncol 2010;5(6):909 - 16. http://dx.doi.org/10. 1097/JTO.0b013e3181d89b48. 